\title{
The StockingCost Constraint
}

\author{
Vinasétan Ratheil Houndji, Pierre Schaus, Laurence Wolsey and Yves Deville \\ Université catholique de Louvain, Louvain-la-Neuve, Belgium \\ \{vinasetan.houndji, pierre.schaus, laurence.wolsey, \\ yves.deville\}@uclouvain.be
}

\begin{abstract}
Many production planning problems call for the minimization of stocking/storage costs. This paper introduces a new global constraint StockingCost $\left(\left[X_{1}, \ldots, X_{n}\right],\left[d_{1}, \ldots, d_{n}\right], H, c\right)$ that holds when each item $X_{i}$ is produced on or before its due date $d_{i}$, the capacity $c$ of the machine is respected, and $H$ is an upper bound on the stocking cost. We propose a linear time algorithm to achieve bound consistency on the StockingCost constraint. On a version of the Discrete Lot Sizing Problem, we demonstrate experimentally the pruning and time efficiency of our algorithm compared to other state-of-the-art approaches.
\end{abstract}

Keywords: Production Planning, Discrete Lot Sizing, Constraint Programming, Global Constraint

\section{Introduction}

Production planning problems, such as Lot Sizing and Scheduling Problems, require one to determine a minimum cost production schedule to satisfy the demands for single or multiple items without exceeding machine capacities while satisfying demands. Reviews of those problems and the corresponding Mixed Integer Programming (MIP) formulations are presented in [8|2|5|12]. In many Lot Sizing and Scheduling problems, in particular when the planning horizon is discrete and finite, there are stocking costs to minimize. These costs depend on the time spent between the production of an item and its delivery (due date).

To handle such Lot Sizing Problems in Constraint Programming, we propose an efficient bound consistency filtering algorithm for the StockingCost $\left(\left[X_{1}, \ldots, X_{n}\right],\left[d_{1}, \ldots, d_{n}\right], H, c\right)$ constraint that requires each item $X_{i}$ to be produced on or before its due date $d_{i}$ and the capacity $c$ of the machine to be respected.

First, we define the StockingCost constraint and how one can achieve pruning with the state-of-the-art approaches. After, we present some algorithms to achieve Bound Consistency for the total stocking costs $H$ and for the items $X_{i}, i \in[1 . . n]$. Then, we propose a complete $O(n)$ filtering algorithm to achieve Bound Consistency for all variables. Finally, we present some experimental results on a Lot Sizing Problem and conclude. 


\section{The StockingCost Constraint}

The StockingCost constraint has the following form:

$$
\text { StockingCost }\left(\left[X_{1}, \ldots, X_{n}\right],\left[d_{1}, \ldots, d_{n}\right], H, c\right)
$$

where

- the variable $X_{i}$ is the date of production of item $i$ on the machine,

- the integer $d_{i}$ is the due-date for item $i$,

- the integer $c$ is the maximum number of items the machine can produce during one time slot (capacity),

- if an item is produced before its due date, then it must be stocked. The variable $H$ is an upper bound on the total number of slots all the items are need in stock.

The StockingCost constraint holds when each item is produced before its due date $\left(X_{i} \leq d_{i}\right)$, the capacity of the machine is respected (i.e. no more than $c$ variables $X_{i}$ have the same value), and $H$ is an upper bound on the total stocking $\operatorname{cost}\left(\sum_{i}\left(d_{i}-X_{i}\right) \leq H\right)$.

Definition 1. Each variable has a finite domain. We denote by $X_{i}^{\text {min }}$ and $H^{\text {min }}$ (resp. $X_{i}^{\max }$ and $H^{\max }$ ) the minimal (resp. maximal) value in the domain of variable $X_{i}$ and $H$. We also denote $t_{\max }=\left(\max _{i}\left(X_{i}^{\max }\right)-\min _{i}\left(X_{i}^{\min }\right)\right)$.

Note that StockingCost can be viewed as a soft-constraint 13 that would impose every item to be produced exactly at the deadline. The stocking cost variable $H$ is the violation of these deadlines. We use inequality instead of equality (as in van-Hoeve's definition of soft-constraints [13]) because StockingCost is an optimization constraint with $H$ typically minimized. Observe that it differs from a standard inequality constraint mainly because $H^{\max }$ (the value representing the current best solution) will change during the search for a solution 13. In particular, it implies that we can filter the domains of variables $X_{i}$ with respect to $H^{\max }$, and potentially increase $H^{\min }$ with respect to $X_{i}$ 's.

The objective of a filtering algorithm is to remove values that do not participate in any solution of the constraint. In this paper, we are interested in achieving bound-consistency for the StockingCost constraint. This consistency level generally offers a good trade-off between speed and filtering power. In a bound consistent constraint, every variable bound (maximum or minimum) occurs in a solution of the constraint. More formally, the bound-consistency definitions for the StockingCost constraint are:

Definition 2. Given a domain $\mathcal{D}$ of variables $X_{i}$ and $H$, the constraint StockingCost $\left(\left[X_{1}, \ldots, X_{n}\right],\left[d_{1}, \ldots, d_{n}\right], H, c\right)$ is bound consistent with respect to $\mathcal{D}$ iff

$$
\begin{aligned}
- & \mathbf{B C}\left(X_{i}^{\min }\right)(1 \leq i \leq n) \text { Let } x_{i}=X_{i}^{\min } ; \text { there exist } x_{j} \in \\
& {\left[X_{j}^{\min } . . X_{j}^{\max }\right](1 \leq j \leq n, i \neq j) \text { and } h=H^{\max } \text { such that } } \\
& \text { StockingCost }\left(\left[x_{1}, \ldots, x_{n}\right],\left[d_{1}, \ldots, d_{n}\right], h, c\right) \text { holds }
\end{aligned}
$$




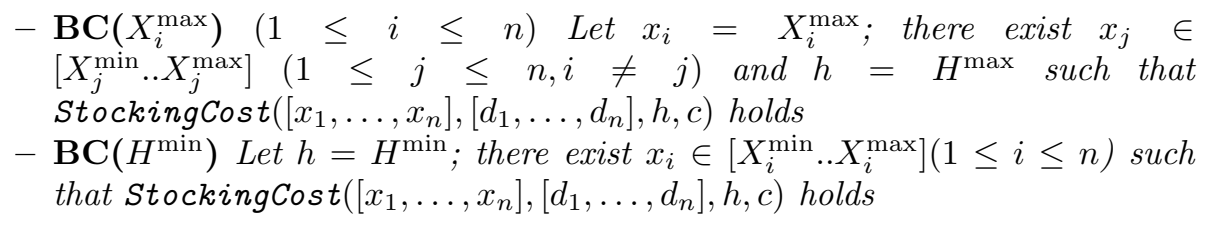

\section{Decomposing the constraint}

It is classical to decompose a global constraint into a conjunction of simpler constraints, and applying the filtering algorithms available on the simpler constraints. This raises two questions. First, does the filtering on the decomposition achieves bound consistency? Second, if it achieves the same filtering, what is the complexity of this filtering?

A first decomposition of the constraint StockingCost $\left(\left[x_{1}, \ldots, x_{n}\right],\left[d_{1}, \ldots, d_{n}\right], h, c\right)$ is the following:

$$
\begin{array}{r}
X_{i} \leq d_{i}, \forall i \\
\sum_{i}\left(X_{i}=t\right) \leq c, \forall t \\
\sum_{i}\left(d_{i}-X_{i}\right) \leq H
\end{array}
$$

Assuming that the filtering algorithms for each of the separate constraints achieve bound consistency, the above decomposition does not achieve bound consistency of the StockingCost constraint, as illustrated in the following example.

Example 1. Consider the following instance StockingCost $\left(\left[X_{1} \in[1 . .2], X_{2} \in\right.\right.$ $\left.[1 . .2]],\left[d_{1}=2, d_{2}=2\right], H \in[0 . .2], c=1\right)$. The naive decomposition is not able to increase the lower bound on $H$ because the computation of $H$ gives $\left(2-X_{1}\right)+$ $\left(2-X_{2}\right)=[0 . .1]+[0 . .1]=[0 . .2]$. The problem is that it implicitly assumes that both items can be placed at the due date but this is not possible because of the capacity 1 of the machine. The lower bound of $H$ should be set to 1 . It corresponds to one item produced in period 1 and the other in period 2 .

Other decompositions can be proposed to improve the filtering of the naive decomposition.

A first improvement is to use the global cardinality constraint (gcc) to model the capacity requirement of the machine imposing that no value should occur more than $c$ times. The $g c c$ constraint can efficiently replace $t_{\max }$ constraints of equation 2 in the basic decomposition. Bound consistency on the gcc constraint can be obtained in $O(n)$ plus the time for sorting the $n$ variables [9. However, together with equation 3 , they do not achieve bound consistency of StockingCost constraint.

A second possible improvement is to use a cost based global cardinality constraint (cost-gcc) [10]. In the cost-gcc, the cost of the $\operatorname{arc}\left(X_{i}, v\right)$ is equal 
to $+\infty$ if $v>d_{i}$ and $d_{i}-v$ otherwise. The cost - gcc provides more pruning than equations 2 and 3 in the basic decomposition. Enforcing arc-consistency for cost-gcc requires a time complexity of $O(n \cdot S(m, n+d, \gamma))$ to check consistency where $n$ is the number of variables, $d$ is the size of the domains, $m$ is the number arcs and $S(m, n+d, \gamma)$ is the complexity of the search for shortest paths from a node to every node in a graph with $m$ arcs and $n+d$ nodes with a maximal cost $\gamma$ [10. For the StockingCost, there can be up to $n \cdot t_{\max } \operatorname{arcs}$. Hence ${ }^{1}$ the final complexity to obtain arc-consistency ${ }^{2}$ on the cost-gcc used to model StockingCost can be up to $O\left(t_{\max }^{3}\right)$. To the best of our knowledge the arc-consistent cost-gcc constraint has never been implemented in a solver. Note that for $c=1$, one can use a minimum assignment constraint with a filtering based on reduced costs [4. The consistency check for this constraint is achieved in $O\left(t_{\max }^{3}\right)$ (time complexity needed to solve a minimum assignment problem with the Hungarian algorithm). The advantage of the minimum assignment is that a minimum cost assignment can be recomputed in $O\left(\left(t_{\max }\right)^{2}\right)$ for one value removal. It is not possible to clearly characterize the filtering level achieved for the minimum assignment constraint based on reduced-costs.

Without loss of generality, in the rest of paper, we assume that $X_{i}^{\max } \leq d_{i}, \forall i$.

\section{Pruning the cost variable}

Given an assignment/solution $\bar{X}$ on variables $X=\left[X_{1}, \ldots, X_{n}\right]$, we denote by $H(\bar{X})$ the value $\sum_{i}\left(d_{i}-\bar{X}_{i}\right)$.

Observation 1 For two assignments $\bar{X}$ and $\hat{X}$ satisfying $\left|\left\{X_{i}: X_{i}=t\right\}\right| \leq c$, if the sorted sequences of values in these solutions are the same, then $H(\bar{X})=$ $H(\hat{X})$.

Let $\mathcal{P}$ denote the problem of computing the optimal lower-bound for $H$ :

$$
\begin{aligned}
& H^{o p t}(\mathcal{P})=\min \sum_{i}\left(d_{i}-X_{i}\right) \text { s.t. } \\
& X_{i}^{\min } \leq X_{i} \leq X_{i}^{\max }, \forall i \\
& \left|\left\{X_{i}: X_{i}=t\right\}\right| \leq c, \forall t
\end{aligned}
$$

Algorithm 1 computes the optimal value $H^{\text {opt }}(\mathcal{P})$ in $O(n \cdot \log (n))$ and detects infeasibility if the problem not feasible. This algorithm greedily schedules the productions from the latest to the first one. A current time line $t$ is decreased and at each step, all the items such that $X_{i}^{\max }=t$ are stored into a priority queue (heap) to be scheduled next. Note that each item is added/removed exactly once in the heap and the heap is popped at each iteration (line 11). The items with largest $X_{i}^{\text {min }}$ must be scheduled first until no more items can be scheduled in time $t$ or the maximum capacity $c$ is reached.

\footnotetext{
${ }^{1}$ using Fibonacci heap to implement Dijkstra algorithm for shortest path computation ${ }^{2}$ without considering incremental aspects.
} 


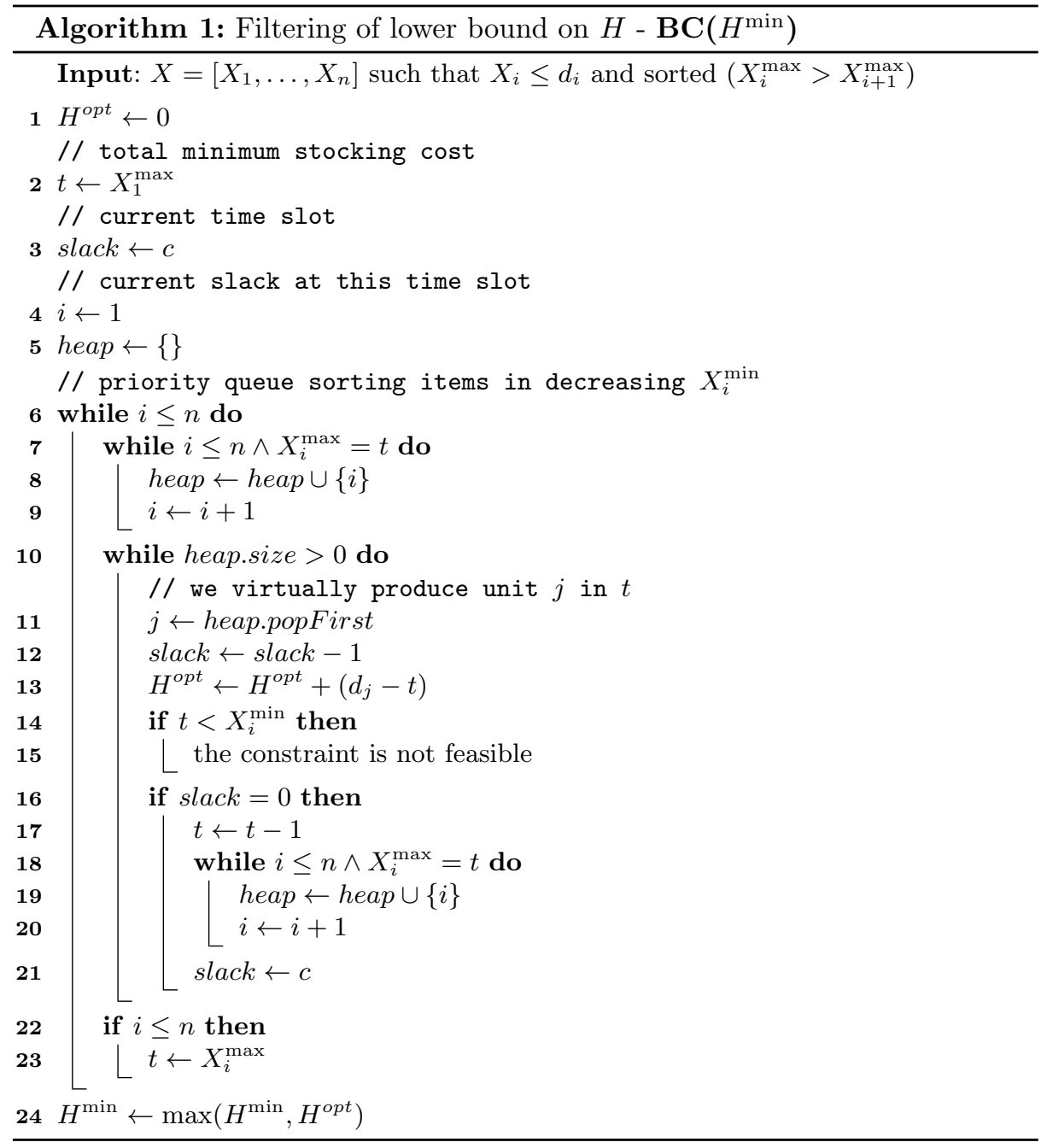

Let $\mathcal{P}^{r}$ denote the same problem with relaxed lower bounds of $X_{i}$ :

$$
\begin{aligned}
& H^{o p t}\left(\mathcal{P}^{r}\right)=\min \sum_{i}\left(d_{i}-X_{i}\right) \text { s.t. } \\
& X_{i} \leq X_{i}^{\max }, \forall i \\
& \left|\left\{X_{i}: X_{i}=t\right\}\right| \leq c, \forall t
\end{aligned}
$$

Observation 2 If problem $\mathcal{P}$ is feasible (i.e. the gcc constraint is feasible), then $H^{\text {opt }}(\mathcal{P})=H^{\text {opt }}\left(\mathcal{P}^{r}\right)$. 
Proof. If we use a simple queue instead of a priority queue in Algorithm 1 , one may virtually assign items to times $t<X_{i}^{\min }$ and the feasibility test is not valid anymore, but the algorithm terminates with the same ordered sequence of time slots used in the final solution. By Observation 1, the objective values of optimal solutions are the same. The complexity of the algorithm without priority queue is $O(n)$ instead of $O(n \cdot \log (n))$.

The greedy Algorithm 1 is able to compute the best lower bound $H^{\text {opt }}\left(\mathcal{P}^{r}\right)$ (in the following we drop problem argument since optimal values are the same) and filters the lower bound of $H$ if possible.

\section{Pruning the item variable}

From now on, since we assume the $g c c$ constraint is already bound-consistent and thus feasible, only the cost argument may cause a filtering of lower-bounds $X_{i}^{\text {min }}$. Therefore, in the rest of the article, we implicitly assumed relaxed domains $\left[-\infty . . X_{i}^{\max }\right] \leq d_{i}$.

Definition 3. Let $H_{X_{i} \leftarrow v}^{\text {opt }}$ denote the optimal lower bound in a situation where $X_{i}$ is forced to take the value $v \leq X_{i}^{\max }$.

Clearly, $v$ must be removed from the domain of $X_{i}$ if $H_{X_{i} \leftarrow v}^{o p t}>H^{\max }$. An interesting question is: What is the minimum value $v$ for $X_{i}$ such that $H_{X_{i} \leftarrow v}^{o p t}=$ $H^{o p t}$ ?

Definition 4. Let $v_{i}^{\text {opt }}$ denote the minimum value such that $H_{X_{i} \leftarrow v}^{\text {opt }}=H^{\text {opt }}$. We have $v_{i}^{o p t}=\min \left\{v \leq X_{i}^{\text {max }}: H_{X_{i} \leftarrow v}^{\text {opt }}=H^{\text {opt }}\right\}$.

The following observation gives a lower bound on the evolution on $H^{o p t}$ when a variable $X_{i}$ is forced to take a value $v<v_{i}^{o p t}$.

Observation 3 For $v<v_{i}^{o p t}$, we have $H_{X_{i} \leftarrow v}^{o p t} \geq H^{o p t}+\left(v_{i}^{o p t}-v\right)$

After the propagation of $H^{\text {min }}$, one may still have some slack between the upper and the lower bound $H^{\max }-H^{\min }$. Since $v_{i}^{o p t}$ is the minimum value such that $H_{X_{i} \leftarrow v}^{o p t}=H^{o p t}$, we can use the lower bound of Observation 3 to filter $X_{i}$ as follows:

$$
X_{i}^{\min } \leftarrow \max \left(X_{i}^{\min }, v_{i}^{o p t}-\left(H^{\max }-H^{\min }\right)\right)
$$

In the following we show that the lower-bound of Observation 3 can be improved and that we can actually predict the exact evolution of $H_{X_{i} \leftarrow v}^{o p t}$ for an arbitrary value $v<v_{i}^{o p t}$. A valuable information to this end is the number of items scheduled at a given time slot $t$ in an optimal solution:

Definition 5. In an optimal solution $\bar{X}$ (i.e. $H(\bar{X})=H^{\text {opt }}$ ), let

$$
\operatorname{count}[t]=\left|\left\{i: \bar{X}_{i}=t\right\}\right| .
$$


Algorithm 2 computes $v_{i}^{o p t}, \forall i$ and count $[t], \forall t$ in linear time $O\left(t_{\max }\right)$. The first step of the algorithm is to initialize count $[t]$ as the number of variables with upper bound equal to $t$. This can be done in linear time assuming the time horizon of size $\left(\max _{i}\left\{X_{i}^{\max }\right\}-\min _{i}\left\{X_{i}^{\min }\right\}\right)$ is in $O(n)$. We can initialize an array count of the size of the horizon and increment the entry count $\left[X_{i}^{\max }\right]$ of the array in $O(1)$ for each variable $X_{i}$.

The idea of the Algorithm 2 is to use a Disjoint-Set $T$ (also called unionfind) data structure [1 making it possible to have efficient operations for T.Union $\left(S_{1}, S_{2}\right)$, grouping two disjoint sets into a same set, and T.Find $(v)$ returning a "representative" of the set containing $v$. It is easy to extend a disjointset data structure with operations T.min $(v) / T \cdot \max (v)$ returning the minimum/maximum value of the set containing value $v$. As detailed in the invariant of the algorithm, time slots are grouped into a set $S$ such that if $X_{i}^{\max } \in S$ then $v_{i}^{o p t}=\min S$.

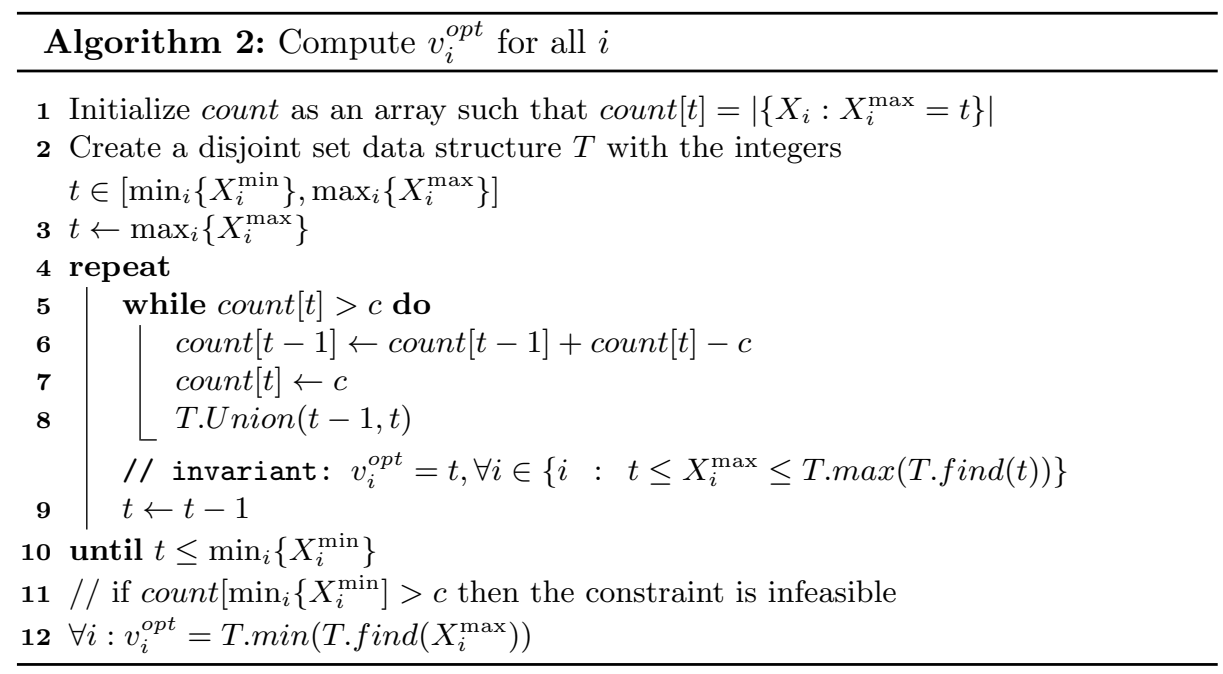

Example 2. Consider the following instance StockingCost $\left(\left[X_{1} \in[1 . .3], X_{2} \in\right.\right.$ $\left.[1 . .6], X_{3} \in[1 . .7], X_{4} \in[1 . .7], X_{5} \in[1 . .8]\right],\left[d_{1}=3, d_{2}=6, d_{3}=7, d_{4}=\right.$ $\left.\left.7, d_{5}=8\right], H \in[0 . .4], c=1\right)$. At the beginning of the algorithm, count $=$ $[0,0,1,0,0,1,2,1]$ and $T=\{\{1\},\{2\},\{3\},\{4\},\{5\},\{6\},\{7\},\{8\}\}$. After the loop, count $=[0,0,1,0,1,1,1,1]$ and $T=\{\{1\},\{2\},\{3\},\{4\},\{5,6,7\},\{8\}\}$. Thus $v_{X_{1}}^{o p t}=3, v_{X_{2}}^{o p t}=v_{X_{3}}^{o p t}=v_{X_{4}}^{o p t}=5$ and $v_{X_{5}}^{o p t}=8$. Next figure shows the different steps of the computation of $T$. Note that from step 3 to step 8 , there is no change since for $t \in[1 . .6]$, count $[t]$ always $\leq 1$ in the loop.

Observation 3 gives a lower bound on the evolution of the optimal stocking cost when assigning variable $X_{i}$ to $v$. Unfortunately, this lower bound is not 


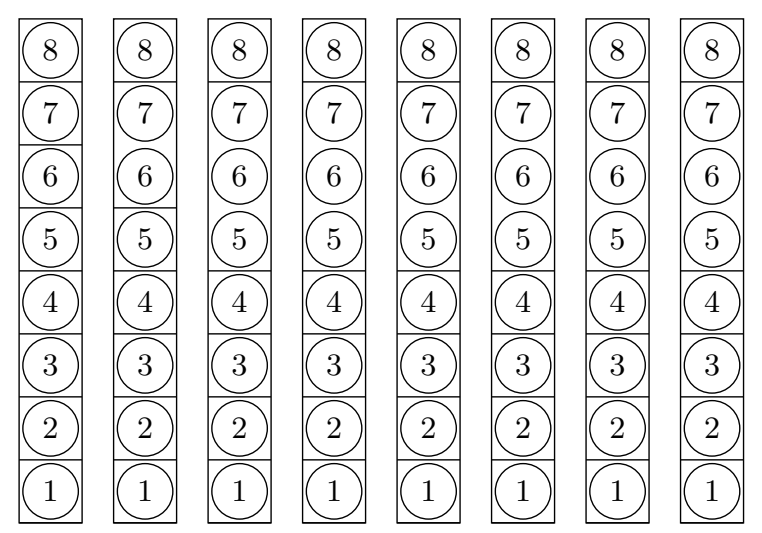

step 1 step 2 step 3 step 4 step 5 step 6 step 7 step 8 $\mathrm{t}=8 \mathrm{t}=7 \mathrm{t}=6 \mathrm{t}=5 \mathrm{t}=4 \mathrm{t}=3 \mathrm{t}=2 \mathrm{t}=1$

optimal. One can be convinced easily for instance that with $c=1$, if $v<v_{i}^{\text {opt }}$ is assigned $X_{i}$, it virtually imposes to move to left at least all variables $X_{j}$ such that $\left\{X_{j}: X_{j}^{\max }=v\right\}$. This suggests for $\mathrm{c}=1$, the following improved lower bound for $H_{X_{i} \leftarrow v}^{o p t}$ :

$$
H_{X_{i} \leftarrow v}^{o p t} \geq H^{o p t}+\left(v_{i}^{o p t}-v\right)+\left|\left\{X_{j}: X_{j}^{\text {max }}=v\right\}\right|
$$

Next example illustrates that this lower bound is still not optimal. It is not sufficient just only consider the set $\left\{X_{j}: X_{j}^{\max }=v\right\}$ since more variables could be impacted.

Example 3. Consider the following instance StockingCost $\left(\left[X_{1} \in[1 . .5], X_{2} \in\right.\right.$ [1..4], $\left.\left.X_{3} \in[1 . .4]\right],\left[d_{1}=5, d_{2}=4, d_{3}=4\right], H \in[0 . .10], c=1\right)$ with $H^{o p t}=1$ and $v_{X_{1}}^{o p t}=5$. For $v=4, H_{X_{1} \leftarrow 4}^{o p t} \geq H^{o p t}+\left(v_{X_{1}}^{o p t}-v\right)+\left|\left\{X_{j}: X_{j}^{\max }=v\right\}\right|=$ $1+(5-4)+2=4$. Here, $H_{X_{1} \leftarrow 4}^{o p t}$ is really 4 . For $v=3, H_{X_{1} \leftarrow 3}^{o p t} \geq H^{o p t}+\left(v_{X_{1}}^{o p t}-\right.$ $v)+\left|\left\{X_{j}: X_{j}^{\max }=v\right\}\right|=1+(5-3)+0=3$ but here $H_{X_{1} \leftarrow 3}^{\text {opt }}=4$.

Definition 6. A slot $t$ is full if it is using all its capacity count $[t]=c$.

Observation 4 There is at most $\left\lfloor\frac{n}{c}\right\rfloor$ full time slots.

Definition 7. minfull $[t]$ is largest time slot $\leq t$ which is not full. More exactly $\operatorname{minfull}[t]=\max \left\{t^{\prime} \leq t: \operatorname{count}\left[t^{\prime}\right]<c\right\}$.

Definition 8. $\operatorname{maxfull}[t]$ is the smallest time slot $\geq t$ which is not full. More exactly $\max f u l l[t]=\min \left\{t^{\prime} \geq t: \operatorname{count}\left[t^{\prime}\right]<c\right\}$.

Next observation gives the exact evolution of $H_{X_{i} \leftarrow v}^{o p t}$ that will allow the BC filtering of $X_{i}^{\min }$.

Observation $5 H_{X_{i} \leftarrow v}^{o p t}=H^{o p t}+\left(v_{i}^{o p t}-v\right)+(v-\operatorname{minf} u l l[v]), \forall v<v_{i}^{o p t}$ 
To understand the previous observation one can realize that the number of variables affected (that would need to be shifted by one to the left) by assigning $X_{i} \leftarrow v$ is equivalent to the impact caused by insertion of an artificial item with the domain $[-\infty . . v]$. So, the exact impact of $X_{i} \leftarrow v$ is the number of variables affected by the move plus $\left(v-v_{X_{i}}^{o p t}\right)$. The Algorithm 3 computes $\operatorname{minfull}[t], \max f u l l[t], \forall t$. The time complexity is thus $O\left(t_{\max }\right)$.

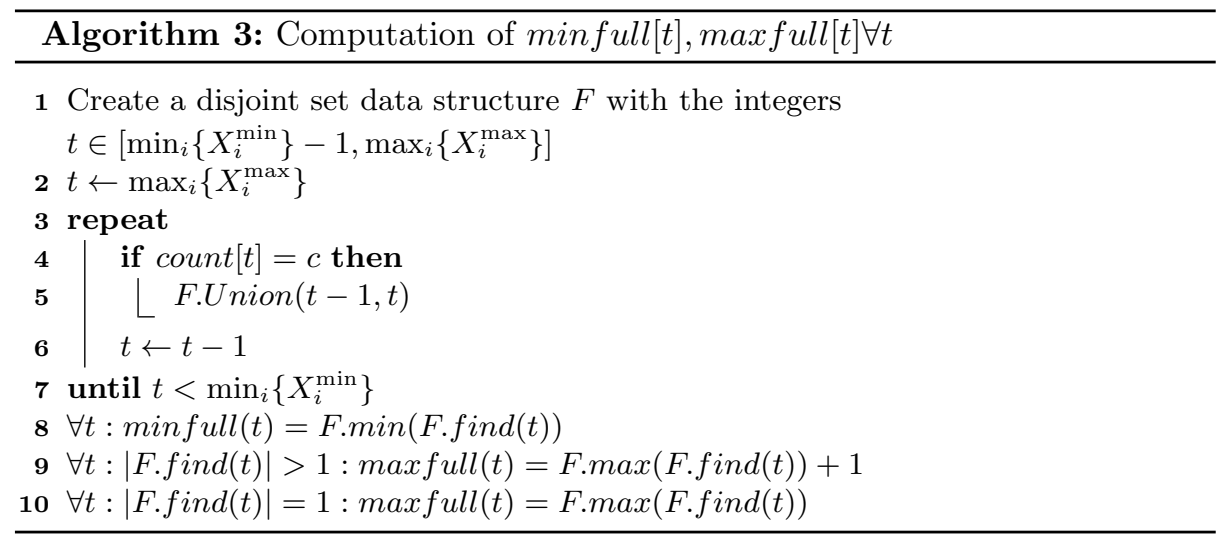

Observation $6 H_{X_{i} \leftarrow t}^{o p t} \geq H_{X_{i} \leftarrow t^{\prime}}^{o p t} \forall t<t^{\prime} \leq v_{i}^{o p t}$

Observation 7 If a slot $t$ is full $(\operatorname{count}[t]=c)$ then $\forall i$ :

$$
H_{X_{i} \leftarrow t}^{\text {opt }}=H_{X_{i} \leftarrow t^{\prime}}^{\text {opt }}, \forall t^{\prime} \in[\operatorname{minfull}[t] . . \max f u l l[t]) \text { such that } t^{\prime}<v_{i}^{\text {opt }}
$$

Proof. Suppose that a slot $t$ is full. We know that $\forall t^{\prime} \in[\operatorname{minfull}[t]$. max $f u l l[t])$, $\operatorname{minfull}\left[t^{\prime}\right]=\operatorname{minfull}[t]$. Thus, $\forall t^{\prime} \in[\operatorname{minfull}[t] . . \max f u l l[t])$ such that $t^{\prime}<$ $v_{i}^{o p t}, H_{X_{i} \leftarrow t^{\prime}}^{\text {opt }}=H^{\text {opt }}+\left(v_{i}^{o p t}-t^{\prime}\right)+\left(t^{\prime}-\operatorname{minfull}\left[t^{\prime}\right]\right)=H^{\text {opt }}+v_{i}^{o p t}-\operatorname{minfull}[t]=$ $H^{o p t}+\left(v_{i}^{o p t}-t\right)+(t-\operatorname{minfull}[t])=H_{X_{i} \leftarrow t}^{o p t} . \square$

The above observation is very important because if the new minimum for $X_{i}$ falls on a full time slot, we can increase the lower bound further. The bound consistent filtering rule is given in Algorithm 4 .

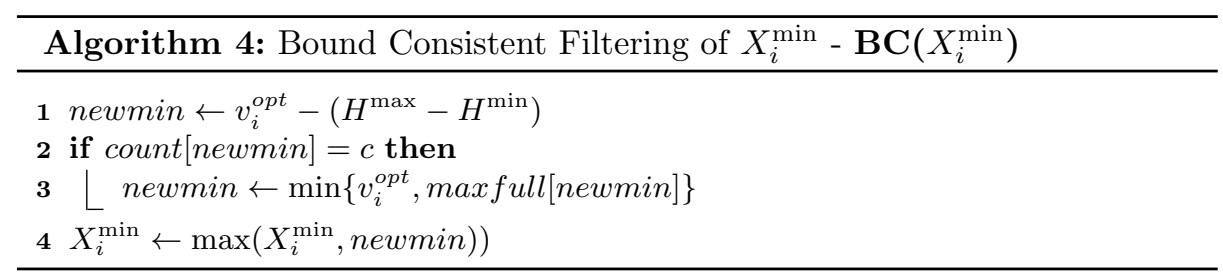


Example 4. Considering the following instance StockingCost $\left(\left[X_{1} \in[1 . .3], X_{2} \in\right.\right.$ $\left.[1 . .6], X_{3} \in[1 . .7], X_{4} \in[1 . .7], X_{5} \in[1 . .8]\right],\left[d_{1}=3, d_{2}=6, d_{3}=7, d_{4}=7, d_{5}=\right.$ $8], H \in[0 . .4], c=1)$. We know that $v_{X_{1}}^{\text {opt }}=3, v_{X_{2}}^{\text {opt }}=v_{X_{3}}^{\text {opt }}=v_{X_{4}}^{o p t}=5, v_{X_{5}}^{\text {opt }}=8$ and count $=[0,0,1,0,1,1,1,1]$. After running the algorithm 11 we have $H^{\text {opt }}=$ 2 and thus $H \in[2 . .4]$. Algorithm 3 gives $F=\{\{0\},\{1\},\{2,3\},\{4,5,6,7,8\}\}$,

minfull $=[1,2,2,4,4,4,4,4]$ and $\max f u l l=[1,2,4,4,9,9,9,9]$. Algorithm 4 gives for:

- $X_{1}:$ newmin $=3-2=1$. count $[1]=0$ and $X_{1}^{\min }=\max \{1,1\}=1$;

$-X_{2}, X_{3}, X_{4}:$ newmin $=5-2=3$. count $[3]=1$, newmin $=\min \{4,5\}=4$ and $X_{j \in\{2,3,4\}}^{\min }=\max \{1,4\}=4$. Next figure shows the evolution of $H_{X_{3} \leftarrow t}^{\text {opt }}$. Note that for $t \in[1 . .3], H_{X_{3} \leftarrow t}^{\text {opt }}>H^{\max }=4$.

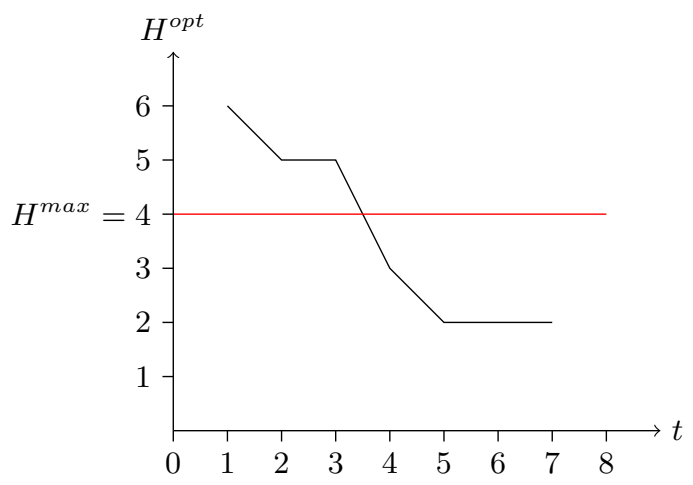

- $X_{5}:$ newmin $=8-2=6$. count $[6]=1$, newmin $=\min \{8,9\}=8$ and $X_{5}^{\min }=\max \{1,8\}=8$.

Thus $X_{1} \in[1 . .3], X_{2} \in[4 . .6], X_{3} \in[4 . .7], X_{4} \in[4 . .7]$ and $X_{5} \in\{8\}$.

\section{A complete filtering algorithm in $O(n)$}

The Algorithms 2 and 3 for computing $v_{i}^{o p t}, \forall i$ and $\max f u l l(t), \forall t$ presented so far have a complexity of $O\left(t_{\max }\right)$. Although for some problems $t_{\max } \approx n$, in practice it can be larger than $n$ if there is some sparsity on the deadlines. Algorithm 5 describes a complete self-contained version of the filtering for StockingCost running in $O(n)$ given a sorted version of the variables. This algorithm keeps tracks of the items in the same set (same $v^{o p t}$ ) by maintaining two indexes $j, k$ with the following properties:

- After line 10, items in $\{j, \ldots, i\}$ are the open items $\left(X_{i}: X_{i}^{\max } \geq t\right)$ that still need to be placed into some slots in an optimal solution.

- After line 10, all the items in $\{k, \ldots, i\}$ have the same $v^{\text {opt }}$. This value $v^{\text {opt }}$ is only known when all the current remaining open items can be placed into the current slot. That is when the condition at line 13 is true. 
Variable $u$ keeps track of the maxfull( $t)$ potential value with maxfull $(t)$ implemented as a map with constant time insertion. Only time slots $t$ with $\max f u l l(t)>t$ are added to the map. Each time a full slot $t$ is discovered (at lines 19 and 26), one entry is added to the map. By observation 4 the number of entries added into the map is at most $n$.

Lines 29 to 34 are just applying the filtering rules from Algorithm 4

Implementation Details Although the Algorithm 5 is in $O(n)$, it requires the variables to be sorted. Since the filtering algorithms are called multiple times during the search process and only a few number of variables are modified between each call, simple sorting algorithms such as insertion or bubble sort are generally more efficient than classical sorting algorithms $O(n \cdot \log (n))$.

The map can be a simple Hashmap but a simple implementation with two arrays of size $t_{\max }$ and a magic number incremented at each call can be used to avoid computing hash functions and the map object creation/initialization at each call to the algorithm. One array contains the value for each key index in the map, and the other array contains magic numbers containing the value of the magic number at the insertion. An entry is present only if the value at corresponding index in the magic array is equal to the current magic number. Incrementing the magic number thus amounts at emptying the map in $O(1)$. The cost $O\left(t_{\max }\right)$ at the map creation has to be paid only once an is thus amortized.

\section{$6 \quad$ Experimental results}

Experiments were conducted on instances $M I-D L S-C C-S C$ (Multi Item - Discrete Lot Sizing - Constant Capacity - Setup Cost) problems described in 8.

\section{Description of the $M I-D L S-C C-S C$ problem}

The Discrete Lot Sizing problem considered here is a multi-item, single machine problem with capacity of production limited to one per period. There are storage costs and sequence-dependent changeover costs, respecting the triangle inequality. Each order consisting of one unit of a particular item has a due date and must be produced at latest by its due date. The stocking (inventory) cost of an order is proportional to the number of periods between the due date and the production period. The changeover cost $q^{i, j}$ is induced when passing from the production of item $i$ to another one $j$ with $q^{i, i}=0 \forall i$. Backlogging is not allowed. The objective is to assign a production period for each order respecting its due date and the machine capacity constraint so as to minimize the sum of stocking costs and changeover costs.

Next example shows a tiny instance of the problem.

Example 5. Consider the problem with the following input data: number of items type nbItems $=2$; number of periods nbPeriods $=5$; stocking cost $h=2$; 


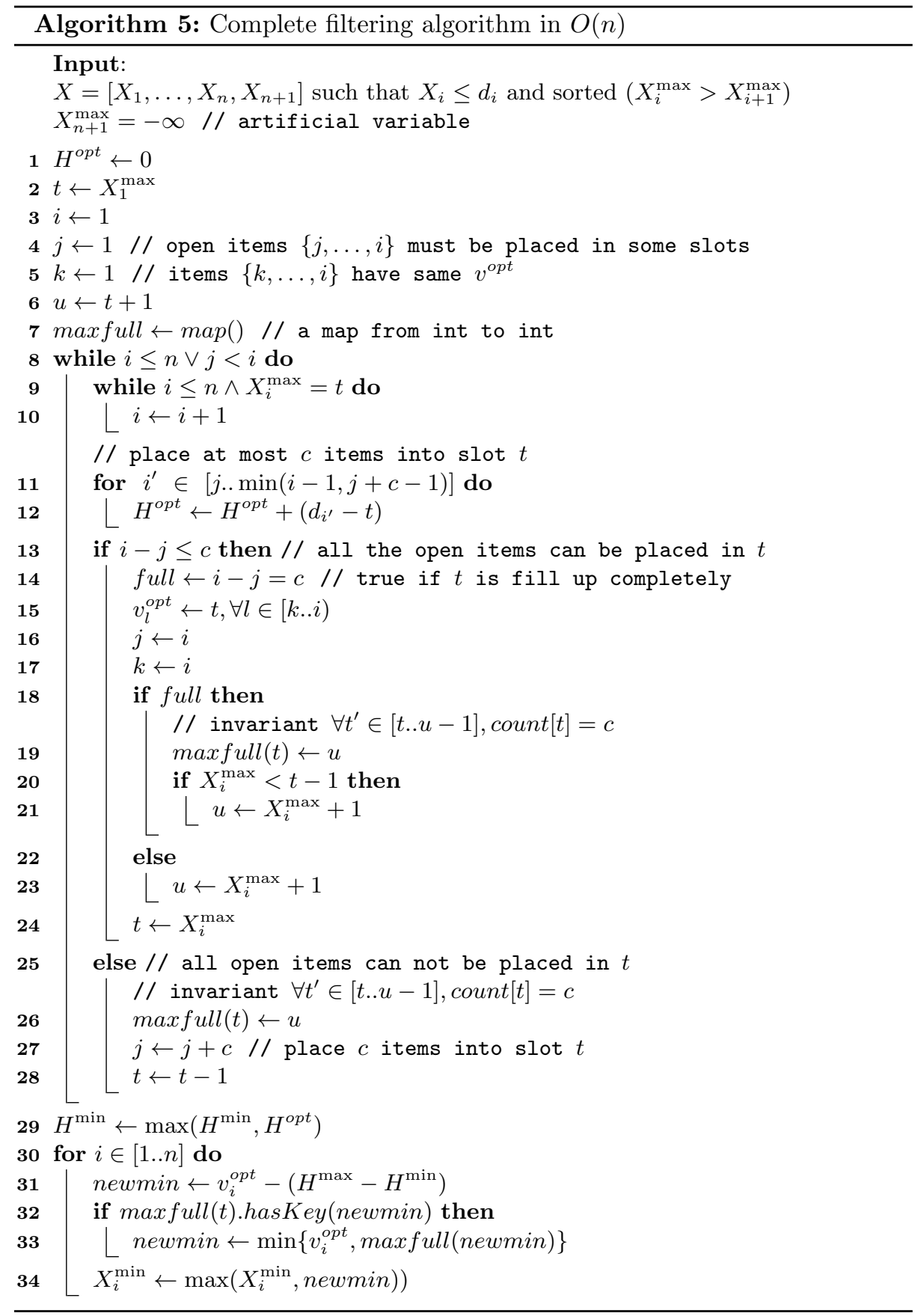


demand times for items of type $1 d_{t \in\{1, \ldots, 5\}}^{1}=(0,1,0,0,1)$ and for items of type $2 d_{t \in\{1, \ldots, 5\}}^{2}=(1,0,0,0,1) ; q^{1,2}=5, q^{2,1}=3$. A feasible solution of this problem is productionPlan $=(2,1,2,0,1)$ which means that item 2 will be produced in period 1; item 1 in period 2; item 2 in period 3 and item 1 in period 5 . Note that there is no production in period 4 , it is an idle period. The cost associated to this solution is $q^{2,1}+q^{1,2}+q^{2,1}+2 * h=15$ but it is not the optimal cost. The optimal solution is productionPlan $=(2,1,0,1,2)$ with the cost $q^{2,1}+q^{1,2}+h=10$.

\section{A Constraint Programming Model}

We uniquely identify each order. The aim is to associate to each of these orders a period that respects the due date of the order. Let date $(p) \in[1 . . n b$ Periods $]$, $\forall p \in[1 . . n b$ Demands], represents the period in which the order $p$ is satisfied. This corresponds to period in which the order $p$ is produced/satisfied. Let dueDate $(p)$ be the deadline for order $p$, that is the period in which $p$ is due.

If objStorage is an upper bound on the total number of periods in which orders have to be held in stock, the stocking part can be modeled by the constraint:

$$
\text { StockingCost(date, dueDate, objStorage, 1) }
$$

Observation 8 There is no difference between two orders of the same item except for their due dates. Therefore given a feasible production schedule, if it is possible to swap the production periods of two orders involving the same item same item (date $\left(p_{1}\right)$, date $\left(p_{2}\right)$ such that item $\left(p_{1}\right)=$ item $\left.\left(p_{2}\right)\right)$, we obtain an identical solution with the same stocking cost..

Based on observation 8, we remove such symmetries by adding precedence constraints on date variables involving by the same item:

$$
\begin{gathered}
\operatorname{date}\left(p_{1}\right)<\operatorname{date}\left(p_{2}\right), \forall\left(p_{1}, p_{2}\right) \in[1 . . n b D e m a n d s] \times[1 . . n b D e m a n d s] \text { such that } \\
\text { dueDate }\left(p_{1}\right)<\operatorname{dueDate}\left(p_{2}\right) \wedge \operatorname{item}\left(p_{1}\right)=\operatorname{item}\left(p_{2}\right)
\end{gathered}
$$

Now, the second part of the objective objChangeover concerning changeover costs has to be introduced in the model. This part is similar to a successor CP model for the Traveling Salesman Problem (TSP) in which the cities to be visited represent the orders and the distances between them are the corresponding changeover costs. Let successor $(p), \forall p \in[1 . . n b D e m a n d s]$, define the order produced on the machine immediately after producing order $p$. We additionally create a dummy order nbDemands +1 to be produced after all the other orders. In the first step, a Hamiltonian circuit successor variable is imposed. This is achieved by using the classical circuit [7] constraint on successor variables for dynamic subtour filtering. The date and successor variables are linked with the element constraint by imposing that the production date of $p$ is before the production date of its successors:

$$
\forall p \in[1 . . n b \text { Demands }]: \operatorname{date}(p)<\operatorname{date}(\operatorname{successor}(p))
$$


As announced, the artificial production is scheduled at the end:

$$
\text { date }(n b \text { Demands }+1)=\text { nbPeriods }+1
$$

Note that as with date variables, some symmetries can be broken. For two nodes $n_{1}, n_{2} \in[1 . . n b D e m a n d s]$ such that dueDate $\left(n_{1}\right)<\operatorname{dueDate}\left(n_{2}\right)$ and item $\left(n_{1}\right)=\operatorname{item}\left(n_{2}\right)$, we force that $n_{1}$ cannot be the successor of $n_{2}$ with successor $\left(n_{2}\right) \neq n_{1}$. Finally, a minAsssignment constraint 3 is used on the successor variables and the changeover part of the objective objChangeover.

The objective to minimize is simply the sum of stocking costs and changeover costs : (objStorage $* h)+$ objChangeover, where $h$ is the unit stocking cost.

\section{Experimental results}

By assuming that the basic filtering $\operatorname{date}(p) \leq \operatorname{dueDate}(p), \forall p \in$ [1..nbDemands] is imposed a priori, we compare the performance of the filtering algorithm due to StockingCost (date, deadline, objStorage, 1) constraint with that achieved by the following three sets of constraints:

- the basic decomposition :

$$
\begin{aligned}
& \sum_{p}(\operatorname{date}(p)=t) \leq 1, \forall t \in[1 . . n b \text { Periods }] \\
& \sum_{p}(\operatorname{dueDate}(p)-\operatorname{date}(p)) \leq \text { objStorage }
\end{aligned}
$$

- $t_{\max }$ constraints of the previous decomposition are replaced by the global bound consistency constraint allDifferentBC [6], that is the special case of $g c c$ constraint when the capacity $c=1$ :

$$
\begin{array}{r}
\text { allDifferentBC(date }) \\
\sum_{p}(\text { dueDate }(p)-\operatorname{date}(p)) \leq \text { objStorage }
\end{array}
$$

- the global constraint minAssignment $[\underline{3}$ on date and objStorage variables is added to the previous decomposition.

The StockingCost filtering algorithm and the $M I-D L S-C C-S C$ model have been implemented in the OscaR open-source solver [11. They will be available in OscaR from release 1.1.0. As search heuristic, we used a classical static binary search on date and successor variables in order to reduce the impact of the search on model comparisons. Table 1 shows the results for some randomly generated instances of $M I-D L S-C C-S C{ }^{3}$ We present, for each group of constraints, the number of nodes visited and the time (in seconds) used to complete the search.

\footnotetext{
${ }^{3}$ Instances available at http://becool.info.ucl.ac.be/resources/ 


\begin{tabular}{|c|c|c|c|c|c|c|c|c|}
\hline \multirow[t]{2}{*}{ Instance } & \multirow{2}{*}{\multicolumn{2}{|c|}{$\begin{array}{l}\text { StockingCost } \\
\text { Nodes Time }\end{array}$}} & \multirow{2}{*}{\multicolumn{2}{|c|}{$\begin{array}{l}\text { Minassignment } \\
\text { Nodes Time }\end{array}$}} & \multirow{2}{*}{\multicolumn{2}{|c|}{$\begin{array}{l}\text { AllDifferent } \\
\text { Nodes Time }\end{array}$}} & \multicolumn{2}{|c|}{ Basic decomp } \\
\hline & & & & & & & Nodes & Time \\
\hline 1(15_5_13) & $0.3610^{6}$ & 26 & $0.4110^{6}$ & 36 & $1.2510^{6}$ & 87 & $1.2510^{6}$ & 99 \\
\hline $2\left(15 \_5 \_14\right)$ & $0.9810^{6}$ & 79 & $1.2610^{6}$ & 112 & $3.1610^{6}$ & 255 & $3.1710^{6}$ & 283 \\
\hline 3(15_8_13) & $1.1010^{6}$ & 64 & $2.3410^{6}$ & 156 & $8.0510^{6}$ & 515 & $8.0710^{6}$ & 625 \\
\hline 4(15_10_12) & $0.2210^{6}$ & 12 & $0.7210^{6}$ & 32 & $8.0210^{6}$ & 385 & $8.1010^{6}$ & 478 \\
\hline 5(15_10_14) & $\mathbf{0 . 3 2} 10^{6}$ & 16 & $1.4110^{6}$ & 79 & $18.710^{6}$ & 1350 & $18.810^{6}$ & 1552 \\
\hline $6\left(20 \_5 \_17\right)$ & $\mathbf{1 . 1 4} 10^{6}$ & 135 & $1.4010^{6}$ & 213 & $3.3310^{6}$ & 353 & $4.0710^{6}$ & 548 \\
\hline 7(20_10_18) & $6.9010^{6}$ & 534 & $8.0210^{6}$ & 805 & $9.6810^{6}$ & 906 & - & 一 \\
\hline 8(20_10_19) & $\mathbf{1 . 3 2} 10^{6}$ & 95 & $1.3410^{6}$ & 120 & $9.6810^{6}$ & 616 & - & - \\
\hline $9\left(30 \_5 \_12\right)$ & $\mathbf{2 . 8 7} 10^{6}$ & 124 & $3.0010^{6}$ & 223 & $3.0010^{6}$ & 127 & $3.0010^{6}$ & 188 \\
\hline 10(30_10_11) & $\mathbf{5 . 5 1} 10^{6}$ & 244 & $6.6810^{6}$ & 530 & $7.7310^{6}$ & 342 & $7.7310^{6}$ & 494 \\
\hline 11(30_10_16) & $2.4110^{6}$ & 156 & $4.6410^{6}$ & 439 & - & - & - & - \\
\hline 12(100_10_11) & $1.4910^{6}$ & 60 & $1.5010^{6}$ & 271 & $2.3010^{6}$ & 110 & $2.3010^{6}$ & 153 \\
\hline 13(100_10_18) & $0.1110^{6}$ & 10 & $0.1510^{6}$ & 63 & $0.3610^{6}$ & 23 & $2.9610^{6}$ & 331 \\
\hline 14(100_15_17) & $2.7910^{6}$ & 143 & $6.5110^{6}$ & 1132 & $22.210^{6}$ & 1305 & $22.310^{6}$ & 1712 \\
\hline 15(200_15_22) & $19.310^{6}$ & 854 & - & - & $24.610^{6}$ & 1187 & $24.610^{6}$ & 2024 \\
\hline
\end{tabular}

Table 1. Results for 15 MI-DLS-CC-SC instances. The format of instance is the following: InstanceNumber(nbPeriods_nbItems_nbDemands). "-_" means that the model did not complete the search after 3600 seconds.

These results suggest that our StockingCost version offers a stronger and faster filtering than other decompositions. In particular, the last four instances suggest that the time complexity of our filtering algorithm scales better than the minAssignment decomposition when the number of time slots increases. This is not surprising since filtering algorithm for StockingCost is in $O$ (nbDemands) and not a function of the size of horizon as is the case for the minAssignment decomposition.

\section{Conclusion}

In this paper, we have introduced a new global constraint StockingCost to handle the stocking aspect of Lot Sizing Problems when using Constraint Programming. We have described an advanced filtering algorithm achieving bound consistency with a time complexity linear in the number of variables. The experimental results show the pruning and time efficiency of the StockingCost constraint on a version of the Discrete Lot Sizing Problem compared to various decompositions of the constraint.

\section{References}

1. Thomas H Cormen, Charles E Leiserson, Ronald L Rivest, and Clifford Stein. Introduction to Algorithms (Second ed.), chapter 21: Data structures for Disjoint 
Sets. MIT press Cambridge, 2001.

2. A Drexl and A Kimms. Lot sizing and scheduling - survey and extensions. European Journal of Operational Research, pages 221-235, 1997.

3. Filippo Focacci, Andrea Lodi, and Michela Milano. Cost-based domain filtering. In Principles and Practice of Constraint Programming-CP'99, pages 189-203. Springer, 1999.

4. Filippo Focacci, Andrea Lodi, Michela Milano, and Daniele Vigo. Solving tsp through the integration of or and cp techniques. Electronic notes in discrete mathematics, 1:13-25, 1999.

5. Raf Jans and Zeger Degraeve. Modeling industrial lot sizing problems: A review. International Journal of Production Research, 2006.

6. Alejandro López-Ortiz, Claude-Guy Quimper, John Tromp, and Peter van Beek. A fast and simple algorithm for bounds consistency of the alldifferent constraint. In International Joint Conference on Artificial Intelligence - IJCAI03, 2003.

7. Gilles Pesant, Michel Gendreau, Jean-Yves Potvin, and Jean-Marc Rousseau. An exact constraint logic programming algorithm for the traveling salesman problem with time windows. Transportation Science, 32(1):12-29, 1998.

8. Yves Pochet and Laurence Wolsey. Production Planning by Mixed Integer Programming. Springer, 2005.

9. Claude-Guy Quimper, Peter Van Beek, Alejandro López-Ortiz, Alexander Golynski, and Sayyed Bashir Sadjad. An efficient bounds consistency algorithm for the global cardinality constraint. In Principles and Practice of Constraint Programming-CP 2003, pages 600-614. Springer, 2003.

10. Jean-Charles Régin. Cost-based arc consistency for global cardinality constraints. Constraints, 7(3-4):387-405, 2002.

11. OscaR Team. Oscar: Scala in or. https://bitbucket.org/oscarlib/oscar, 2014.

12. Hafiz Ullah and Sultana Parveen. A literature review on inventory lot sizing problems. Global Journal of Researches in Engineering, 10:21-36, 2010.

13. Willem-Jan van Hoeve. Over-constrained problems. In Hybrid Optimization, pages 191-225. Springer, 2011. 